\title{
10
}

\section{Multicopy ARQ Strategies for Heterogeneous Networks}

\author{
M. Aghadavoodi Jolfaei, U. Quernheim \\ Department of Computer Science (Info IV), Aachen University of Technology \\ Ahornstraße 55, D-52074 Aachen, Germany \\ Tel.: +4924180 21413, Fax: +492418888220 \\ E-mail: masoud@informatik.rwth-aachen.de
}

\begin{abstract}
On systems consisting of packet switched networks (e.g. LANs, HSLANs and ATM/BISDN) which are linked over satellite, there are packet losses due to buffer overflow at bridges and routers as well as bit errors on the satellite link. The resulting high error probability requires error correction methods, which are simple and effective in terms of throughput and memory requirements. For packet switched communications, quite a number of ARQ (Automatic Repeat Request) protocols have been designed which are able to cope with stringent memory requirements, but they either lack safety at high block error rates, or they require a large bandwidth due to low throughput. In this paper, we will introduce a simple and effective strategy (called Stutter-XOR or SXOR strategy) to increase throughput of exisiting protocols. In contrast to existing hybrid schemes (combinations of ARQ and forward error correction), it does not only correct bit errors, but also handles block losses. This is achieved by sending additional blocks, so called XOR blocks, which are created by combining other blocks using the XOR (modulo 2 addition) operation. We developed several variants of this strategy. Two of them will be evaluated here by means of analysis and simulation.
\end{abstract}

Keyword Codes: C.2.1; C.2.2

Keywords: Computer-Communication Networks, General; Network Architectures and Design; Network Protocols

\section{INTRODUCTION}

The way in which the retransmissions of erroneous blocks are carried out is the point in which the various ARQ methods differ. With continuous ARQ strategies, the sender continuously transmits new blocks, and the receiver accepts each error-free block and positively acknowledges it by sending an ACK message. On receipt of an erroneous block, the receiver negatively acknowledges the block by sending a NACK. When a NACK arrives at the sender, the transmission of new blocks is interrupted. In case of the Go-Back-N strategy, the sender does not only retransmit the negatively acknowledged block, but also all the following blocks. These are the blocks wich have been transmitted during one round trip delay after the erroneous block. In case of the selective repeat strategy, the sender retransmits only the negatively acknowledged block. These strategies are also used for point-to-multipoint communications. In this case, blocks are considered to be correct (positively acknowledged) if they are correctly received by all receivers. Accordingly, the sender decides about retransmission of a block after receipt of acknowledgements from all $\mathrm{K}$ receivers.

Another way to correct block errors is the use of forward error correction (FEC), which attempts to enable the receiver to correct block errors by appending redundant information to the data blocks. This method can be applied bit-wise as well as to whole blocks. While traditional FEC schemes (such as BCH or Viterbi codes) aim at the correction of bit errors, the 
Stutter-XOR strategy (SXOR strategy) is able to correct whole blocks. This is based on the observation, that in packet switched networks often more packet losses are caused by buffer overflows at bridges, routers and receivers than by bit errors. This is one reason why current transport protocols (such as ISO-OSI-TP4, TCP/IP, XTP) utilize ARQ methods, which correct erroneous blocks by repeating them.

In the last years, few investigations have been done on methods for correcting packet losses [Bier 92], [LaBE 91], [OhKi 91] and these only cope with delay sensitive transmissions (e.g. video or speach transmissions), where the delay caused by repetitions of lost packets is not acceptable. The SXOR strategy can be used as a pure forward error correction method as well as a hybrid scheme in combination with existing ARQ strategies. Here we consider a variant of the stutter (or multicopy) selective repeat strategie, the Weldon scheme, combined with the SXOR strategy.

In this introduction we will first outline the basic communication model we are dealing with. In chapter 2, we will review an existing ARQ protocol, the Weldon strategy [Wel 82]. The SXOR strategy and its applications are introduced in chapter 3 . In chapter 4 , an analysis of the different variants of the SXOR strategy is conducted, the results of which are presented in chapter 5 . Finally, chapter 6 presents an overview of the achieved results.

The time between the transmission of the first bit of the block and the arrival of the corresponding acknowledgement at the sender is called the round trip delay. While the round trip delay of a satellite link remains constant, this may not be the case in other parts of a network, where alternate routing may occur. Here we assume that the round trip delay remains constant for the whole communication path from the sending station to the receiver(s). The round trip delay can also be expressed as the maximum number $\mathrm{S}$ of blocks the sender can transmit until the acknowledgement for the first block arrives. For simplification, we assume an error free feedback channel (with a propagation delay equal to that of the satellite link) for the acknowledgements, i.e. the acknowledgements do not contribute to the link load and are received correctly after one round trip time.

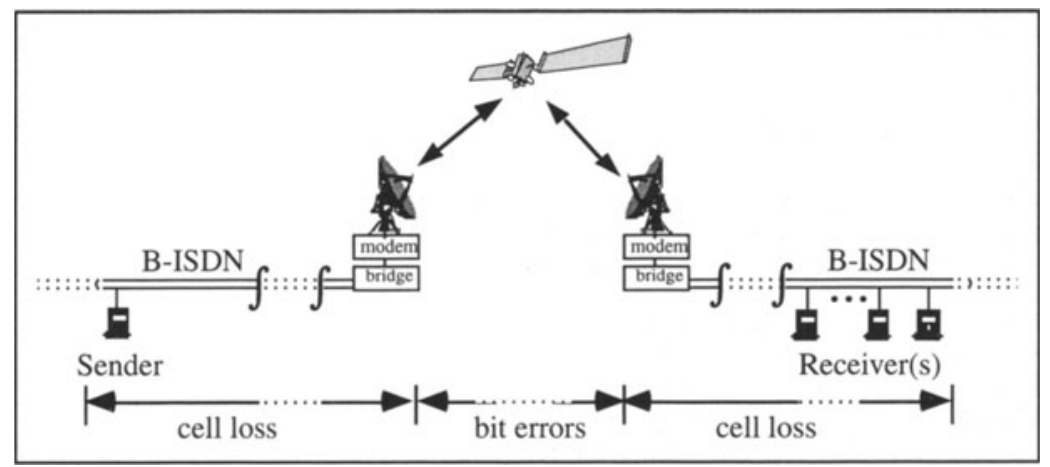

Figure 1: Connecting B-ISDN networks via satellite.

The transmitted blocks are safeguarded by a a set of parity bits, e. g. CRC (Cyclic Redundancy Check). The CRC bits enable the receiver to detect transmission errors. A block error occurs when there is at least one bit error in the block. In this case, the CRC bits do not match the data bits of the block. Undetected block errors are not considered. It is assumed that block errors occur independently of each other [AgKM 92]. A block is affected by an error with probability $\mathrm{p}$, and unaffected with 1-p. Given a bit error probability of $\mathrm{p}_{\mathrm{b}}$, and with 
independent bit errors the resulting block error rate is $\mathrm{p}=1-\left(1-\mathrm{p}_{\mathrm{b}}\right)^{\mathrm{L}}$, where $\mathrm{L}$ is the block length in bits.

As a scenario we consider a heterogenous network consisting of high speed and satellite links (see figure 1). While on the terrestrial links cell losses may occur, the satellite link is dominated by bit errors. We assume that in case of an ATM network a block consists of k cells. A block is said to be erroneous, if it has at least one cell missing or contains at least one erroneous bit.

\section{WELDON STRATEGY}

In [Wel 82] , E.J. Weldon proposes a more advanced multicopy scheme. It is assumed that the receiver has a limited buffer capacity of $\mathrm{q} \cdot \mathrm{S}$ blocks, where $\mathrm{S}$ again denotes the maximum number of blocks which may be sent during one round trip time and $q$ is a fixed integer. This means that there is room for $\mathrm{q}$ complete round trip times in the receiver buffer. When the sender receives a NACK for a specific block, it retransmits this block not just once but $n_{1} \geq 1$ times. In case that each of the retransmitted blocks is disturbed again, the sender now retransmits the block $n_{2}>n_{1}$ times and so on, up to $n_{q}$, where $n_{1}, \ldots, n_{q}$ are user determined values. When stage $\mathrm{q}$ is reached, a maximum of $\mathrm{q} \cdot \mathrm{S}$ blocks (new and retransmitted ones) may have been sent and stored in the receiver buffer (due to limited load, retransmissions and erroneous blocks which are discarded, the actual number will be lower) and the sender considers a receiver buffer overflow. Therefore, on further retransmission attempts the sender retransmits $n_{q}$ copies of the disturbed message and one copy of each of the $S$ subsequent messages, which are supposed to have been lost due to the buffer overflow. Figure 2 shows an example with $q=2$, $\mathrm{n}_{1}=2$ and $\mathrm{n}_{2}=3$.

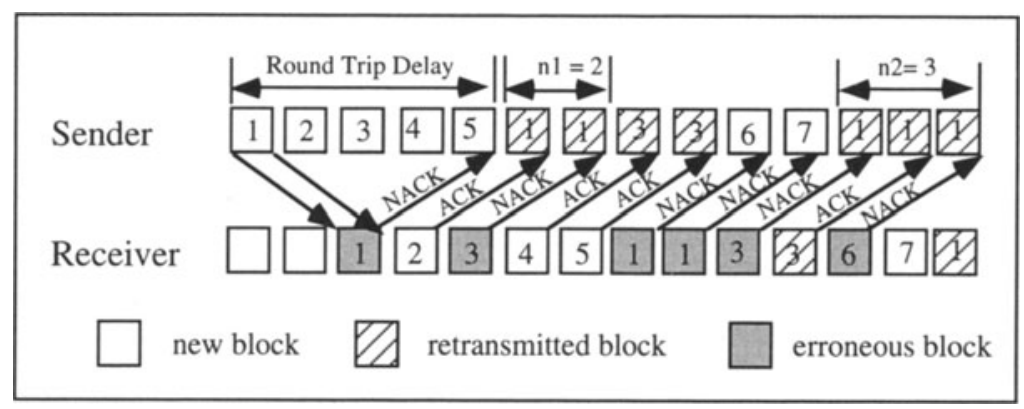

Figure 2: Weldon selective repeat ARQ strategy.

With $\mathrm{q}=1$ and $\mathrm{n}_{1}=1$, the Weldon scheme resembles the ordinary selective repeat scheme. Values larger than $\mathrm{q}=1$ are only useful for excessively high block error rates.

\section{STUTTER-XOR STRATEGY}

The various ARQ schemes differ in the number of retransmissions executed to correct a block error. Multicopy schemes employ a vast number of retransmissions to obtain a high security level, but on the expense of throughput. With our new SXOR strategy, we aim to reduce the number of retransmissions necessary to correct a certain number of block errors using existing ARQ schemes. This is achieved by modulo-2-addition (bit-by-bit logical exclusive OR, $\oplus$ ) of several retransmitted blocks. The strategy works as follows: 
We define a so called XOR window size n. As long as no errors occur, new blocks are transmitted continuously. If, however, one or more NACKs are received at the sending station, it will open an XOR window, in which blocks occur as either new blocks, retransmitted blocks, or XOR blocks, that is, blocks which are modulo-2 sums of the other blocks in the current window. We will term blocks other than XOR blocks basic blocks from now on. The XOR window limits the number basic blocks which can be combined to an XOR block. The receiver which is waiting for a particular block it has not received correctly yet (say, block number B), can retrieve a correct version of B by either waiting for a plain retransmission of B, or by taking advantage of an XOR block which contains B and a number of other blocks it has received correctly.

If, e.g., the receiver has correctly received blocks $\mathrm{A}, \mathrm{C}$, and $\mathrm{D}$, but not $\mathrm{B}$ and $\mathrm{E}$, it may retrieve $\mathrm{B}$ out of the $\mathrm{XOR}$ block $\mathrm{X}=\mathrm{A} \oplus \mathrm{B} \oplus \mathrm{C} \oplus \mathrm{D}$ by calculating $\mathrm{X} \oplus \mathrm{A} \oplus \mathrm{C} \oplus \mathrm{D}$. Substituting $X$ and reordering yields $A \oplus A \oplus C \oplus C \oplus D \oplus D \oplus B=B$, as the sum of a block with itself equals the all zero block. An XOR block containing more than one erroneous blocks cannot be used to correct either one; the block $\mathrm{Y}=\mathrm{B} \oplus \mathrm{C} \oplus \mathrm{E}$ with neither $\mathrm{B}$ nor $\mathrm{E}$ being correctly received would be useless (unless either B or E could be reconstructed from another block). It is important to note that the process of calculating the sum of received blocks can either be conducted on the fly with no extra buffer (compared to non-XOR protocols) by immediately XORing each correctly received block of the XOR window into the receiver input buffer in which incoming channel bits are assembled to blocks, or with one extra buffer especially dedicated to XORing.

\subsection{Stutter-XOR Selective Repeat}

If a NACK is received for a particular block B, the system enters XOR mode for one XOR window. Every group of $\mathrm{n}$ basic blocks is succeeded by an XOR block of the $\mathrm{n}$ basic blocks with the first basic block being the retransmission of $\mathrm{B}$. There may be more retransmission phases (several XOR windows), if more NACKs should occur, just like ordinary selective repeat.

Fig. 3 gives an example for SXOR selective repeat. Let $\mathrm{n}=3$ be the XOR window size, and may the sender have transmitted basic blocks 1, 2, 3, 4 and 5, when it receives a NACK for block 1. Now, an XOR window starts, and $n=3$ blocks are transmitted: first, the retransmission of 1 and new block 6 . A NACK for block 3 arrives at this time and therefore this block is transmitted again rather than new block 7. After this, the window is closed and the

XOR block $X=1 \oplus 6 \oplus 3$ is transmitted. Now, for these four blocks (window and XOR block), there are 16 possible combinations of correct and incorrect blocks. 5 of them (those with 3 or 4 correctly received blocks) yield correct versions of 1,3 , and $6: 3,6$, X correct, 1 , $6, \mathrm{X}$ correct, 1, 3, X correct, 1, 3, 6 correct, and 1,3,6, X correct. For the other 9 combinations (two, one, or no block correct), the XOR block cannot be exploited.

For both ordinary and XOR selective repeat, the exchange of acknowledgements is slightly altered in comparison with ordinary selective repeat. ACKs are emitted immediately upon correct reception, while for NACKs, there are two possible implementations. They are either immediately transmitted, or they are deferred until the end of the window. In the former case, the sender has to deduce from the received acknowledgements, whether or not the receiver has been able to correct the erroneous block. This also means, that there has to be an ACK or NACK for the XOR block. In the latter case, the first NACK is retained until either the XOR window is over (and if the block could be corrected using the XOR block, it is ACKed after all), or until the second NACK. In case of more than one error during one window, the XOR block is useless and erroneous blocks can be NACKed immediately to enable a quicker response by the sender. 


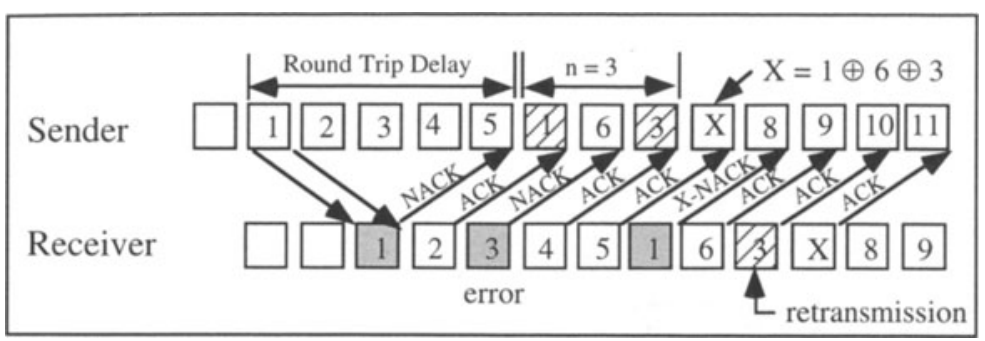

Figure 3: Example for the SXOR selective repeat scheme.

\subsection{Stutter-XOR Weldon}

As with ordinary Weldon, retransmissions occur in q stages, with $n_{i}$ retransmissions on stage i. But now, a complete XOR window rather than a single block is retransmitted $n_{i}$ times. That means, new basic blocks occur during the retransmissions, as well as XOR blocks. An XOR window consists of the block to be retransmitted plus n-1 other basic blocks, succeeded by an XOR block of the whole window (analogous to SXOR selective repeat).

An example for this strategy could look like the one in fig. 4 . We let $n=2$ and $n_{1}=2$ : Blocks $1,2,3,4$ and 5 are transmitted, then a NACK for 1 is received. Stage $\mathrm{q}=1$ is entered, which means that there are $n_{1}=2$ transmissions of an XOR window of length $n=2$.

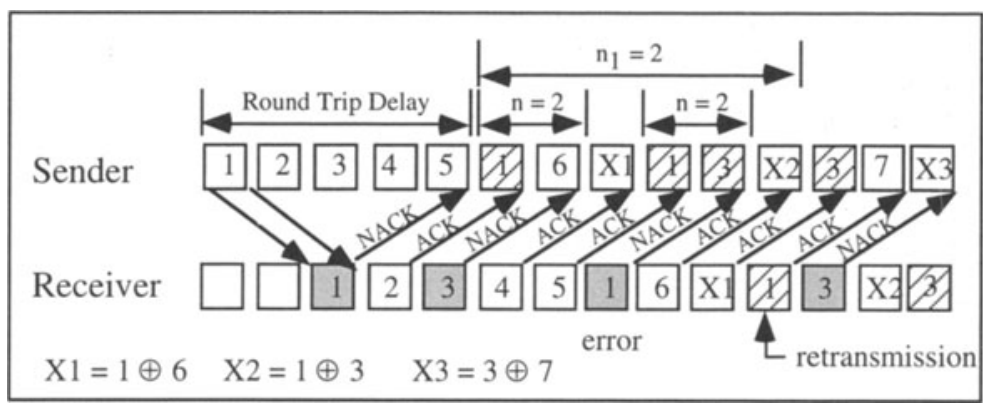

Figure 4: SXOR Weldon scheme.

The first window consists of blocks 1 and 6 , followed by $\mathrm{X} 1=1 \oplus 6$. The second window consists of blocks 1 , and 3, since in the meantime, a NACK for block 3 has been received (otherwise, block 7 would have been sent), succeeded by $X 2=1 \oplus 3$. Stage $q=2$ would yield $\mathrm{n}_{2}$ XOR windows, each with 2 basic blocks and one XOR block of the whole window and so on.

\section{ANALYSIS OF THE SXOR STRATEGY}

For throughput analysis, we have considered two methods, which where presented by Weldon [Wel 82] and Lin/Yu [YuL 81] respectively. As simulations and analytical evaluations have shown, the Weldon method achieves a good approximation of the simulational results despite of its simplicity, but only up to a certain block error probability. The extended Lin/Yu method is more difficult, but approximates the simulational results well even at high block error 
probabilities. In the following we will exemplary demonstrate the Weldon method. We assume that all blocks are of equal length. The additional overhead, which is needed to contain the sequence numbers of the blocks participating an XOR block, is neglected here.

Assuming a saturated sender (no idle times), throughput $\mathrm{T}$ is defined as follows:

$\mathrm{T}=\frac{1}{\mathrm{E}[\mathrm{X}]}$

Here random variable $X$ describes the number of transmissions of a representative block $B$. The expectation value $\mathrm{E}[\mathrm{X}]$ then denotes the mean number of transmission attempts of each block.

Definition (1) must be generalized for our analysis of the SXOR strategy. The reason for this can be demonstrated considering the situation in figure 3 as an example:

\begin{tabular}{llcccc} 
Transmissions & & 6 th & 7 th & 8 th & 9 th \\
Block Numbers & & 1 & 6 & 3 & $X=1 \oplus 6 \oplus 3$ \\
\hline ACK Scenarios & 1) & NACK & ACK & ACK & ACK \\
& 2) & ACK & NACK & ACK & ACK \\
& 3) & ACK & ACK & NACK & ACK
\end{tabular}

Here, for single block errors in the window, block $X$ is required in $1 / 3$ of the cases to either decode basic block 1,3 , or 6 . Therefore, we assign to this block a weight of $1 / 3$ rather than 1 when counting the number of transmissions for each of the three basic block numbers. If we sum up the total weight, three times 1 for the basic blocks and three times $1 / 3$ for block $X$ altogether yield the correct number of 4 block weights. In general, when we are restricting our scope to one representative block B, we assign a weight of $1 / n$ to an XOR block consisting of $n$ basic blocks .

For the analysis we assume a receiver buffer size of S blocks, where is denotes the number of blocks that can be sent during one round trip delay. It is assumed, that at the beginning of the transmission all receiver buffers are empty, and that the probability of block B being correctly received on the first transmission attempt is given by

$\mathrm{P}(\mathrm{X}=1)=1-\mathrm{p}$

In case the first transmission attempt is not successful, $\mathrm{L}$ copies of block B are retransmitted and all correctly transmitted succeeding blocks can be stored in the receiver buffer.The probability, that this transmission turn is successful is

$\mathrm{P}(\mathrm{X}=1+\mathrm{L})=\mathrm{p} \mathrm{Q}(\mathrm{p}, \mathrm{L})$

where $\mathrm{Q}(\mathrm{p}, \mathrm{L})$ is the probability that one of the $\mathrm{L}$ copies of the block is successfully transmitted. The number of blocks sent per repetition is determined by the strategy used. The Weldon strategy simply transmits L copies of the erroneous block. Here, with a probability of $\mathrm{Q}(\mathrm{p}, \mathrm{L})=1-\mathrm{p}^{\mathrm{L}}$ at least one copy is correctly transmitted. With the SXOR-SR strategy, $\mathrm{L}$ is composed of the basic block retransmitted and its share of the XOR block, that is $L=1+\frac{1}{n}$. The probability that block $\mathrm{B}$ can be reconstructed at this attempt is given by

$\mathrm{Q}(\mathrm{p}, \mathrm{L})=(1-\mathrm{p})^{\mathrm{n}+1}+\mathrm{p}(1-\mathrm{p})^{\mathrm{n}}$ 
This is the sum of the probabilities, that either all basic blocks of the SXOR window are being received correctly, or that exactly the observed basic block of the window is erroneous and all others are correct. In a similar manner, terms for $\mathrm{L}$ and $\mathrm{Q}(\mathrm{p}, \mathrm{L})$ of the other strategies are derived.

- Selective Repeat:

$$
\begin{aligned}
& \mathrm{L}=1 \\
& \mathrm{Q}(\mathrm{p}, 1)=1-\mathrm{p}
\end{aligned}
$$

- Weldon $(q=1)$ :

$$
\begin{aligned}
& \mathrm{L}=\mathrm{n}_{1} \\
& \mathrm{Q}\left(\mathrm{p}, \mathrm{n}_{1}\right)=1-\mathrm{p}^{\mathrm{n}_{1}}
\end{aligned}
$$

- SXOR Selective Repeat:

$$
\begin{aligned}
& \mathrm{L}=1+\frac{1}{\mathrm{n}} \\
& \text { QXOR-SR }=\mathrm{Q}\left(\mathrm{p}, 1+\frac{1}{\mathrm{n}}\right)=(1-\mathrm{p})^{\mathrm{n}+1}+\mathrm{p}(1-\mathrm{p})^{\mathrm{n}}
\end{aligned}
$$

- SXOR-Weldon:

$$
\begin{aligned}
& \mathrm{L}=\mathrm{n}_{1}\left(1+\frac{1}{\mathrm{n}}\right) \\
& \mathrm{Q}\left(\mathrm{p}, \mathrm{n}_{1}\left(1+\frac{1}{\mathrm{n}}\right)\right)=1-\left(1-\mathrm{QXOR}_{\mathrm{XR}}\right)^{\mathrm{n}_{1}}
\end{aligned}
$$

If a third transmission attempt is necessary, $1+\mathrm{L}$ blocks have already been transmitted during the first two attempts; now we transmit additional $L+(S-1)$ blocks, because we assume that (S1) new blocks have been sent between the first and second transmission of $B$ and have filled up the receiver buffer. When the third transmission of B arrives at the receiver, (S-1) more blocks have been sent meanwhile, but have been discarded due to buffer overflow, so they also have to be retransmitted. Therefore the probability that this transmission attempt is successful is given by

$$
\mathrm{P}(\mathrm{X}=1+\mathrm{L}+\mathrm{L}+\mathrm{S}-1)=\mathrm{p}(1-\mathrm{Q}(\mathrm{p}, \mathrm{L})) \mathrm{Q}(\mathrm{p}, \mathrm{L})
$$

However, this is a pessimistic assumption: the maximum number of (S-1) blocks are only lost, if $(\mathrm{S}-1)$ blocks have been successfully transmitted. While this assumption works well for low block error probabilities, there is a growing deviation from the real throughput at higher block error probabilities. This is also true at further transmission attempts. For the $\mathrm{m}$-th retransmission $(m \geq 2)$, we get

$\mathrm{P}(\mathrm{X}=1+\mathrm{L}+\mathrm{m}(\mathrm{L}+\mathrm{S}-1))=\mathrm{p}(1-\mathrm{Q}(\mathrm{p}, \mathrm{L}))^{\mathrm{m}-1} \mathrm{Q}(\mathrm{p}, \mathrm{L})$

The sum of the weighted counts yields the expected value of the number of transmission attempts of the representative block $B$ :

$$
\begin{aligned}
\mathrm{E}[\mathrm{X}] & =(1-\mathrm{p})+\sum_{\mathrm{i}=1}^{\infty}(1+\mathrm{L}+(\mathrm{i}-1)(\mathrm{L}+\mathrm{S}-1)) \mathrm{p}(1-\mathrm{Q}(\mathrm{p}, \mathrm{L}))^{\mathrm{i}-1} \mathrm{Q}(\mathrm{p}, \mathrm{L}) \\
& =1-\mathrm{p}(\mathrm{S}-1)+\frac{\mathrm{p}(\mathrm{L}+\mathrm{S}-1)}{\mathrm{Q}(\mathrm{p}, \mathrm{L})}
\end{aligned}
$$

Setting $L=1$ and $Q(p, L)=1-p$ in formula (7) leads to the mean number of transmission attempts needed to correctly transmit a block with the selective repeat strategy [MiSh 81], [Weld 82]: 
$\mathrm{E}[\mathrm{X}]=\frac{1+\mathrm{p}^{2}(\mathrm{~S}-1)}{(1-\mathrm{p})}$

The throughput of the Weldon strategy for $\mathrm{q}=1$ and $\mathrm{n}_{1}$ can be determined by substituting $\mathrm{L}=\mathrm{n}_{1}$ and $\mathrm{Q}\left(\mathrm{p}, \mathrm{n}_{1}\right)=1-\mathrm{p}^{\mathrm{n}_{1}}$. This yields to the result for the Weldon strategy $(\mathrm{q}=1)$ as described in [Weld 82]:

$\mathrm{E}[\mathrm{X}]=1+\mathrm{n}_{1} \mathrm{p}+\frac{\left(\mathrm{n}_{1}+\mathrm{S}-1\right) \mathrm{p}^{1+\mathrm{n}_{1}}}{1-\mathrm{p}^{\mathrm{n}_{1}}}$

\section{RESULTS}

Using the selective repeat strategy, the sender retransmits only negatively acknowledged blocks. The receiver rejects blocks which are either hit by a transmission error or cannot be saved in the receiver buffer. The simulation implements the following method for buffering: Be $\mathrm{i}_{0}$ the oldest block not yet correctly received by the receiver. Then buffer space is reserved for all blocks with a sequence number $\mathrm{i}<\mathrm{i}_{0}+\mathrm{S}$. This space cannot be occupied by newer blocks. In this manner it is assured, that there is room in the buffer when these blocks, which are necessary for conveyance in correct order, are correctly received [YuL 81].

Figures $5 / 6$ and $7 / 8$ show results of analysis and simulation in comparison for point-to-point communication. At high block error probabilities, the analysis is too pessimistic. The simulated system has a lower buffer overflow probability, because erroneous blocks are not being stored in the receiver buffer. With increasing block error rate, this effect becomes predominant, so that analysis and simulation deviate more and more. Because of the growing number of receivers, this tendency is even stronger with point-to-multipoint communications; for this reason, we present only simulational results for this case.

For point-to-point communication and with an XOR window of $n=2$, XOR selective repeat shows the best throughput results above a certain block error probability. A higher window size means that the probability of more than one block error in the window rises. With multiple block errors, the additional XOR block becomes useless, so the results for large window sizes approach those of the selective repeat strategy.

Figures 7 and 8 show a comparison of the selective repeat, Weldon, SXOR selective repeat and SXOR-Weldon strategies for point-to-point communication. Selective repeat achieves the lowest throughput of all schemes. Moreover, the throughput of the SXOR-SR strategy $(n=2)$ is slightly lower than the one achieved by the Weldon strategy $\left(\mathrm{q}=1, \mathrm{n}_{1}=2\right)$. Caused by the higher number of repetitions, the SXOR-Weldon strategy is superior to Weldon and SXOR-SR only for high block error probabilities. Since SXOR-Weldon with $\mathrm{n}_{1}=1$ resembles SXOR-SR, it can be concluded that an adaptive scheme over $\mathrm{n}_{1}$ would achieve the best results.

In contrast to point-to-point communications (fig. 5-8), with a higher number of receivers larger XOR window sizes prove to be useful. When different blocks are correctly received by different receivers, the XOR windows used for repetitions will frequently contain blocks, which have already been correctly received by some receivers at a former transmission attempt. These blocks can be used to decode other blocks from the XOR block, even if they are hit by an error in the current transmission. In this case, even the distortion of more than one block does not make the XOR block useless. On the other side, with a growing XOR window size the overhead caused by the additional XOR blocks is decreased. Figure 9 shows the simulational results of the SR, Weldon and SXOR-SR strategies for point-to-multipoint communications with 50 receivers. 


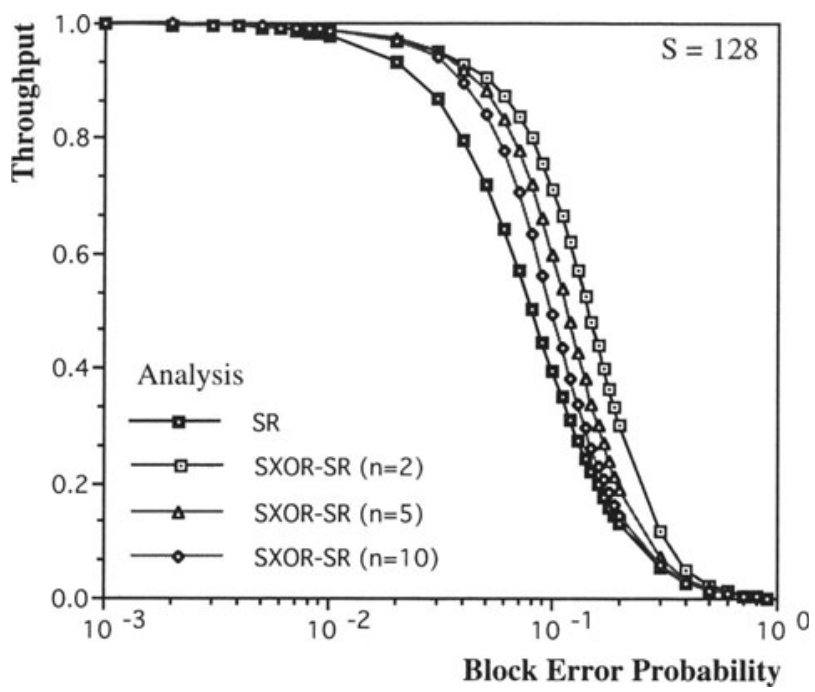

Figure 5: Analysis of the selective repeat and SXOR-SR strategies for various XOR window sizes $(\mathrm{n}=2,5$ and 10 , point-to-point communication )

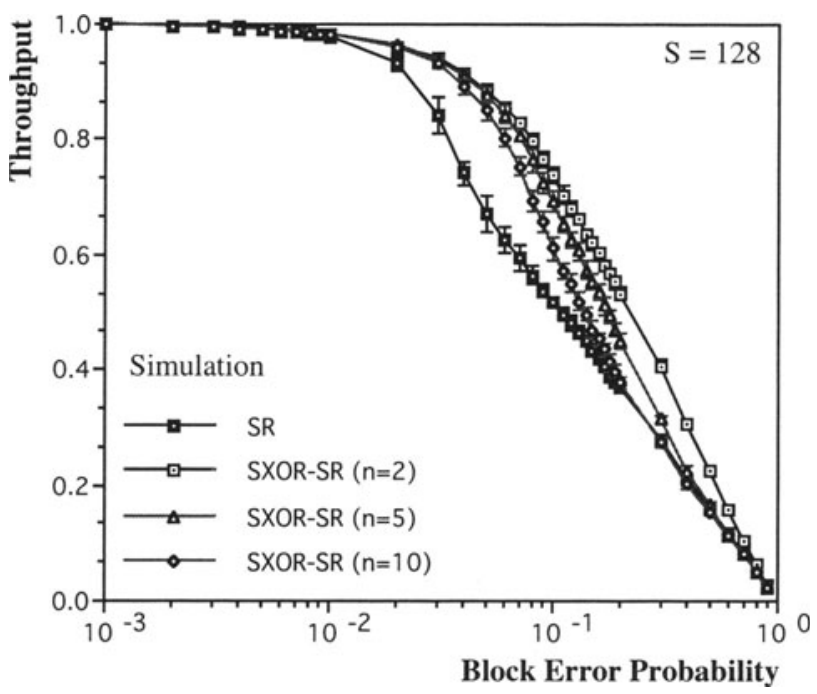

Figure 6: Simulation of the selective repeat and SXOR-SR strategies for various XOR window sizes $(\mathrm{n}=2,5$ and 10, confidence level $99.9 \%)$ 


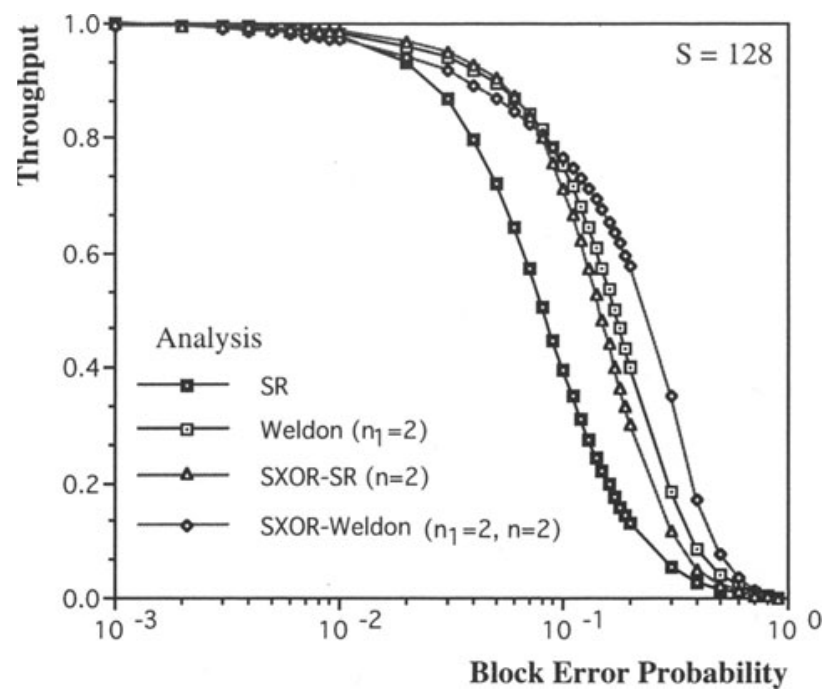

Figure 7: Analytic throughput comparison of selective repeat, Weldon, SXOR-SR and SXOR-Weldon for point-to-point communication

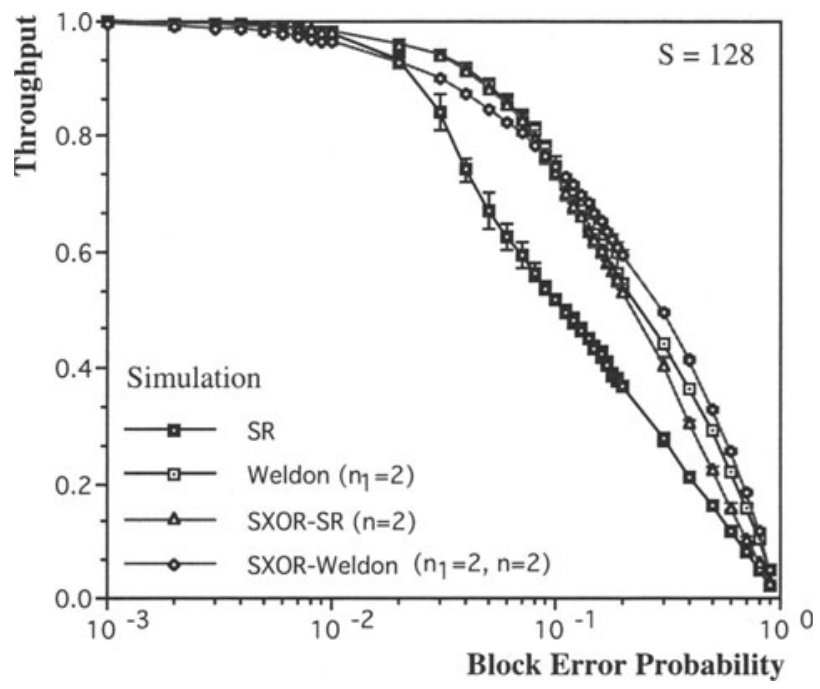

Figure 8: Simulative throughput comparison of selective repeat, Weldon, SXOR-SR and SXOR-Weldon strategies for point-to-point communication (confidence level $99.9 \%$ ) 


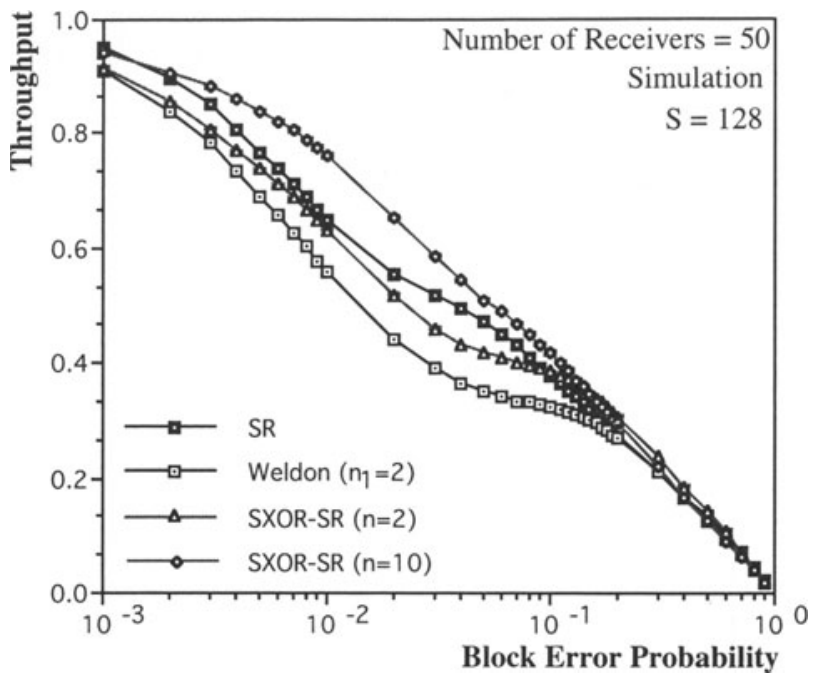

Figure 9: Simulative throughput comparison of selctive repeat, Weldon and SXOR-SR strategies for point-to-multipoint communication (confidence level $99.9 \%$ )

As Figures 10 and 11 demonstrate, with a larger number of receivers multicopy schemes (like Weldon and SXOR Weldon), which transmit a higher number of redundant blocks, show a significantly lower throughput than selective repeat or SXOR-SR.

Figure 11 also shows that at higher block error probabilities and a small number of receivers $(<5)$ the use of Weldon- or SXOR-Weldon is preferable. In this case, an adaptive scheme over $\mathrm{n}_{1}$ and $\mathrm{n}$ would achieve the best results, since SXOR-Weldon with $\mathrm{n}_{1}=1$ resembles SXOR selective repeat.

The SXOR strategy can be used advantageous on channels where both bit errors and packet losses due to buffer overflow occur. This is demonstrated in figure 12, where the results are shown as a function of the bit error probability with an additional constant block loss probability $\mathrm{P}$ (block-loss) of 0.01 . As further simulations have shown, block losses in this range may occur at heavy system load [Naze 92]. Block losses are assumed to be uniformly distributed and independent of each other. The above reviewed strategies can also be combined with traditional FEC schemes. Here we used a $(1023,1013)$ BCH encoding [LiCo 83], which is able to correct 1 bit errors. The FEC encoding is applied to all data blocks, so the number of blocks which can be sent during one round trip delay $(S)$ is slightly decreased $\left(S^{\prime}\right)$. Furthermore, we assume an additional overhead of 8 bits for the SXOR strategy.

Now the throughput is no longer defined in terms of the number of correctly transmitted block per time unit, but in terms of data bits, so that the differing block lengths of the various strategies do not interfere. Throughput efficiency defined as follows:

$\mathrm{T}_{\text {eff }}=\frac{\text { Data (bits) }}{\text { Data (bits) }+ \text { Overhead (bits) }} \frac{1}{\mathrm{E}[\mathrm{X}]}$

As the results show, in this scenario the SXOR-SR scheme is clearly superior to the other strategies, both with and without additional FEC encoding. 


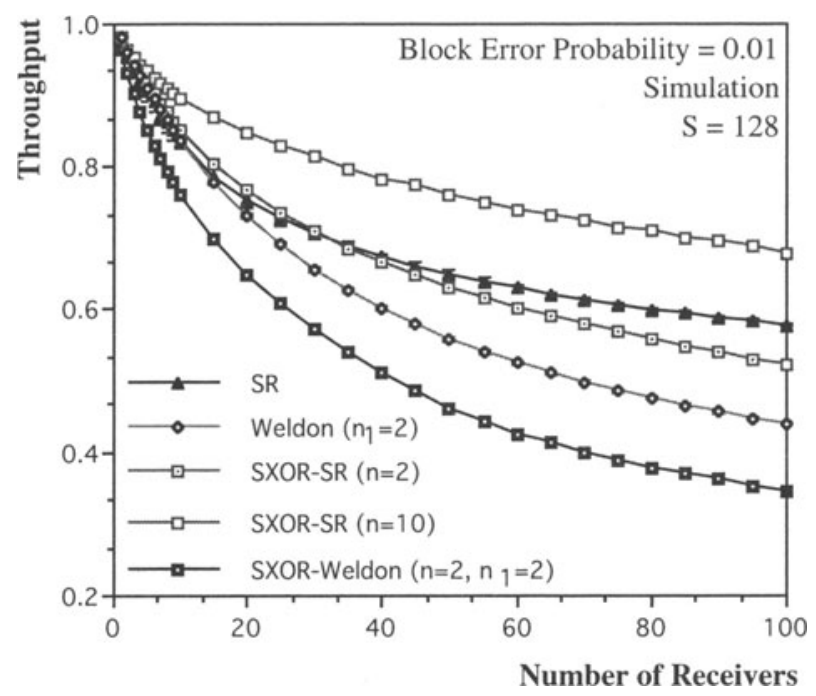

Figure 10: Simulative throughput comparison of the SR, SXOR-SR and SXOR-Weldon strategies for various numbers of receivers (confidence level $99.9 \%$ )

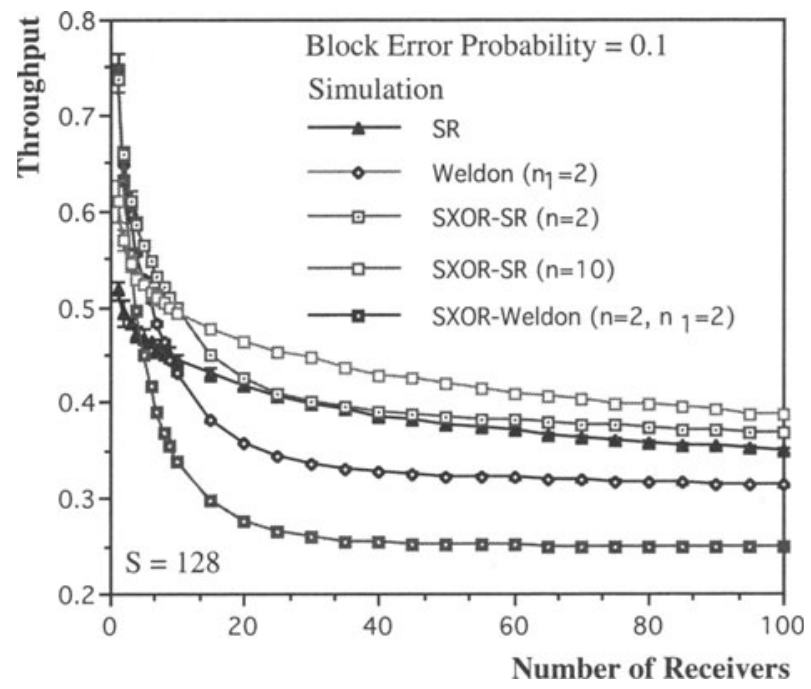

Figure 11: Simulative throughput comparison of the SR, SXOR-SR and SXOR-Weldon strategies for various numbers of receivers (confidence level $99.9 \%$ ) 


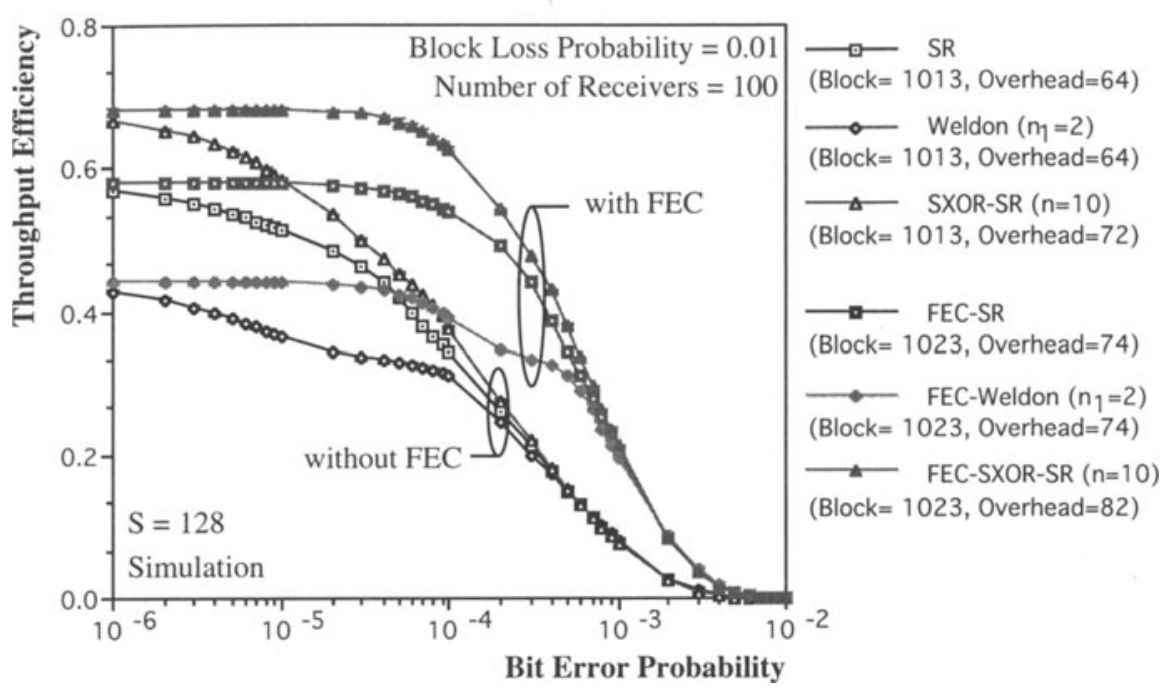

Figure 12: Simulative throughput comparison of the SR, Weldon and SXOR-SR strategies with and without FEC for 100 receivers (confidence level $99.9 \%$ )

\section{CONCLUSION}

We have shown that by combining several blocks into XOR blocks the throughput for existing ARQ protocols can be improved. It can be applied to existing ARQ protocols and will generally yield better performance, especially on transmission channels where both block losses and bit errors occur. For ordinary selective repeat, the improvement is considerable. The Stutter-XOR strategy can even improve the well known Weldon strategy, which has been considered to be most efficient for high block error rates yet. Also, FEC channels can take advantage of XORing. The Stutter-XOR strategy can be implemented very efficiently in software; no fast hardware is required, as for FEC decoders.

\section{REFERENCES}

[AgKM 92] M. Aghadavoodi Jolfaei, D. Kreuer, O. Maly, U. Quernheim: "Two Time Variant Models for Satellite Channels", Proceeding Supercom/ICC 92, Chicago, USA, pp. 314.31.1- 314.3.5.

[AgMM 93] M. Aghadavoodi Jolfaei, S. C. Martin, J. Mattfeldt: "A New Efficient Selective Repeat Protocol for Point-To-Multipoint Communication"; Proceedings ICC '93, pp. 1113-1117, Genf, Swiss, May 93.

[BeF 64] R. J. Benice, Jr. A. H. Frey: "An Analysis of Retransmission System"; IEEE Trans. Commun. Technol., 135-145, Dec. 1964.

[Bier 92] E. W. Biersack: "A Simulation Study of Forward Error Correction in ATM Networks"; Computer Communications Review, 22(1):36-47, January 1992. 
[BrM 86] H. Bruneel, M. Moeneclaey: "On the Throughput Performance of Some Continuos ARQ Strategies with Repeated transmissions"; IEEE Trans, Commun., 244-249, Mar. 1986.

[Bus 72] H. O. Burton, D. D. Sullivan: "Errors and Error Control"; Proc. IEEE, pp. 1293-1303, Nov. 72.

[CaE 86] S. R. Chandran, S. Lin: "A Selective-Repeat ARQ Scheme fot Point-to-Multipoint Communications and It's Throughput Analysis"; Proc. ACM SIGCOM Conference, pp. 292-301, Stowe, VT, Aug. 1984.

[Dav 70] M. A. David: "Order Statistics"; New York: Wiley, 1970.

[GoJ 84] I. S. Gopal, J. M. Jaffe: "Point-to-Multipoint Communication Over Broadcast Links"; IEEE Trans. Commun., Com-3, pp. 1034-1044, Sep. 1984.

[LaBE 91] B. Lamparter, O. Böhrer, W. Effelsberg: "Vorausschauende Fehlerkorrektur für multimediale Datenströme"; GI/ITG-Tagungsband Verteilte Multimedia-Systeme, University Stuttgart, Feb. 1991.

[LiCo 83] S. Lin, D.J. Costello: "Error Control Coding: Fundamentals and Applications"; Prentice Hall, Englewood Cliffs, N.J. 07632, USA, 1983.

[MiSh 81] M. J. Miller, Shu Lin: "The Analysis of Some Selective-Repeat ARQ Schemes with Finite Receiver Buffer"; IEEE Trans. Comm., vol. COM-29, pp. 1307-1315, Sep. 1981.

[Mor 87] J. M. Morris: "On Another Go-Back-N ARQ Technique for High Error Rate Condition"; IEEE Trans. Commun., pp. 187-189, Jan. 1978.

[Naze 93b] M.-R. Nazeman: "Leistungsbewertung unterschiedlicher Protokolle zur Ankopplung von EthernetLANs über Satellit"; Diploma thesis at Aachen University of Technology (Info. IV), June 1993.

[OhKi 91] H. Ohata, T. Kitami: "A Cell Loss Recovery Method Using FEC in ATM Networks"; IEEE Journal on Selected Areas in Communications, Vol. 9, No. 9, pp. 1471-1483, December 1991.

[Sas 75] A. R. K. Sastry: "Improving Automatic Repeat-Request (ARQ) Performance on Satellite Channels under High Error Rate Conditions"; IEEE Trans. Commun. Electron., pp. 224-231, Apr. 1975.

[SaSc 85] K. Sabnani, M. Schwartz: "Multidestination Protocols for Satellite Broadcast Channels"; IEEE Transactions on Communications, Vol. COM-33, No. 3, March 1985.

[Toba 80] F. A. Tobagi: "Multiaccess Protocols in Packet Communication Systems"; IEEE Transactions on Communications, Vol. COM-28, No. 4, pp. 468-488, April 1980.

[ToMi 87] D. Towsley, S. Mithal: "A Selective-Repeat ARQ Protocol for a Point-to-Multipoint Channel"; Proceedings INFOCOM, pp. 521-526, San Franscisco, CA, March 1987.

[Tows 79] D. Towsley: "The Stutter Go-Back-N ARQ Protocol"; IEEE Trans. Comm., vol. COM-27, pp. 869-875, Jun. 1979.

[WaSi 88] J. L. Wang, J. A. Silvester: "Optimal Adaptive ARQ Protocols for Point-to-Multipoint Communication"; IEEE INFOCOM' 88, pp. 704-713, LA, 1988.

[Wel 82] E. J. Weldon: "An Improved Selective Repeat ARQ Strategy"; IEEE Trans. Comm., vol. COM30, pp. 480-486, Mar. 1982.

[YuL 81] P. S. Yu, S. Lin: "An Efficient Selective Repeat ARQ Scheme for Satellite Channels and Its Throughput Analysis"; IEEE Trans. Commun., pp. 353-363, Mar. 1981.

[YuL 82] P. S. Yu, S. Lin: "A Hybrid ARQ Scheme with Parity Retransmission for Error Control of Satellite Channels"; IEEE Transactions on Communications, Vol. COM-30, No. 7, Juli 1982. 